\title{
Application of Ionic Liquid Coating Method to Observation of Non-conductive Samples by a Mobile Scanning Electron Microscope for Elementary Science Education
}

\author{
M. Inoue, ${ }^{1 *}$ M. Suganami, ${ }^{2}$ Y. Hahimoto, ${ }^{3}$ T. Iyasu, ${ }^{3}$ H. Saito, ${ }^{4}$ K. Moriguchi, ${ }^{4}$ and T. Tanaka ${ }^{5}$ \\ ${ }^{I}$ Department of Electrical \& Electronic Engineering, Faculty of Science \& Engineering, \\ Setsunan University, 17-8 Ikeda-nakamachi, Neyagawa, Osaka 572-8508, Japan \\ ${ }^{2}$ Academic Support Center, Setsunan University, 17-8 Ikeda-nakamachi, Neyagawa, Osaka 572-8508, Japan \\ ${ }^{3}$ Shin-nihon Denko Co. Ltd., 4-8-26 Terakawa, Daito, Osaka 574-0014, Japan \\ ${ }^{4}$ APCO Ltd., 522-10 Kitano-machi, Hachioji, Tokyo 192-0906, Japan \\ ${ }^{5}$ Department of Entrepreneur, Graduate School of Engineering, \\ Osaka Sangyo University, 3-1-18 Nakagaito, Daito, Osaka 574-8530, Japan \\ *m-inoue@ele.setsunan.ac.jp
}

(Received : October 3, 2010; Accepted : July 11, 2011)

\begin{abstract}
A battery-powered mobile scanning electron microscope which can be used even in outdoors has been developed for the purpose of showing the mystery of nature and wonder of technology to pupils/students of elementary schools, junior and senior high schools, and so on. We call this compact and easy-operation microscope the MUST-SEM (mobile-use see-through scanning electron microscope). In order to observe non-conductive samples, we employed the ionic liquid coating method. Sample preparation using this method can be carried out easily and rapidly. Observation results of microfossils of Jurassic radiolarian, intertwined genomic DNA fibers extracted from plants, and etc. are reported.
\end{abstract}

\section{Introduction}

Recently, in Japan, the fact that fewer students are majoring in science has become a serious social problem. This is considered to be partly because of the lack of opportunity to observe nature and/or to enjoy scientific experiments in their childhood days. Therefore, many educators have made tremendous efforts to draw pupils' or students' attention to natural science and engineering technologies by demonstrating interesting scientific experiments.

The scanning electron microscope (SEM) is one of the most powerful tool for this purpose because SEM can provide perspective and fascinating images mainly due to its large depth of focus. SEM is, however, generally very expensive as an educational tool, and requires special knowledge and technical training to be able to operate it. Therefore, we have developed a battery-powered mobile and easy-operation SEM which can be used even in outdoors for the purpose of showing the mystery of nature and wonder of technology to pupils/students of elementary schools, junior and senior high schools, and so on. We call this microscope the MUST-SEM (mobile-use see-through SEM) [1].

The surface coating method with conductive materials such as metal or carbon is often employed for the observation of non-conductive samples in order to avoid the charging. In outdoors, however, we can hardly use such vacuum apparatus for coating.

In the present study, we employed the ionic liquid coating method [2,3]. Sample preparation using this method can be performed easily and rapidly, and requires no apparatus for it. Observation results of microfossils of Jurassic radiolarian, intertwined genomic DNA fibers extracted from plants, and etc. are reported. 


\section{Basic Specification of MUST-SEM}

The outer view of the MUST-SEM we developed is shown in Fig.1a. A part of the SEM-column around the electron source is surrounded by a transparent glass cylinder so as to draw students' attention to the inner structure (electron optics). The basic specification is as follows;

1) The MUST-SEM consists of a SEM-column, two vacuum pumps, and a controller (with a LCD display) for them. The weight of the individual part is less than 8 $\mathrm{kg}$ so that elementary school students can carry them in shares with their classmates. The SEM-column is 225 $\mathrm{mm}$ in height and about $2.9 \mathrm{~kg}$ in weight. The weight of the turbo-drag pump and the diaphragm pump are about $1.8 \mathrm{~kg}$, respectively. The weight of the controller without batteries is $7.2 \mathrm{~kg}$, and a battery-pack is $1 \mathrm{~kg}$.

2) The MUST-SEM is a battery-powered system, enabling its operation even in outdoors as shown in Fig.1b. Total electric power demanded to operate the MUST-SEM is about $24 \mathrm{~W}$. We confirmed that the MUST-SEM can be operated for $2 \sim 3$ hours depending on the operational condition with two $24 \mathrm{~V}$-battery-packs, originally made for a motor assisted bicycle. After 5 6 minutes evacuation, the pressure of less than $0.05 \mathrm{~Pa}$ could be achieved.

3) Although the MUST-SEM can also work stand-alone, it can be connected with a personal computer to display the current status of MUST-SEM, or to save image data. Moreover, it can be connected with an extra-display, or a projector so that a lot of students can enjoy change in images on a large display or screen during SEM operation at the same time.

4) The electron optics of the MUST-SEM consists of two electrostatic lenses (condenser and objective), a set of 4-pole electrostatic double deflector and an objective aperture of $100 \mu \mathrm{m}$ diameter. Both of the secondary electrons and the back-scattered electrons are collected as the current signal by a metal plate located in front of the objective lens as shown in Fig.1a. The minimum resolution is estimated to be about $1.7 \mu \mathrm{m}$.

\section{Ionic Liquid Coating Method}

There are some techniques to avoid the charging in the SEM observation of non-conductive samples as follows;

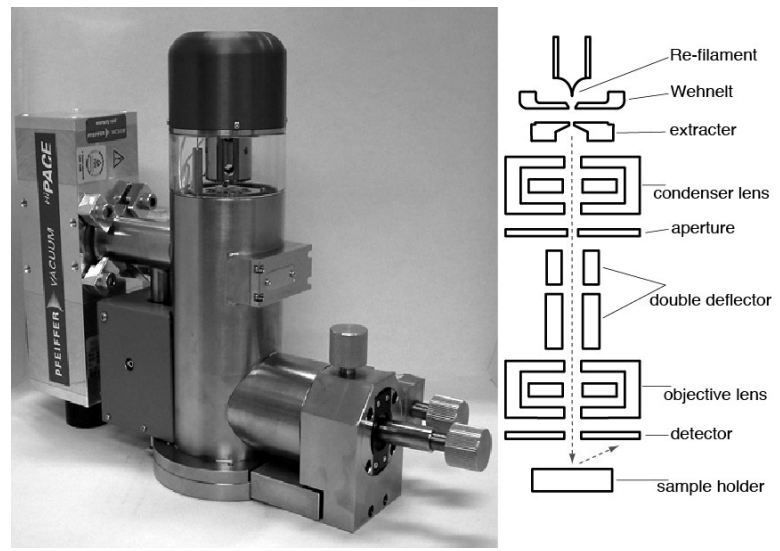

Fig. 1a The outer view of the MUST-SEM with the schematic drawing of the electron optics. The SEM-column is $225 \mathrm{~mm}$ in height and about $2.9 \mathrm{~kg}$ in weight. It is just about the size of $500 \mathrm{ml}$ PET bottle. A part of the column around the filament is surrounded by a transparent glass cylinder.

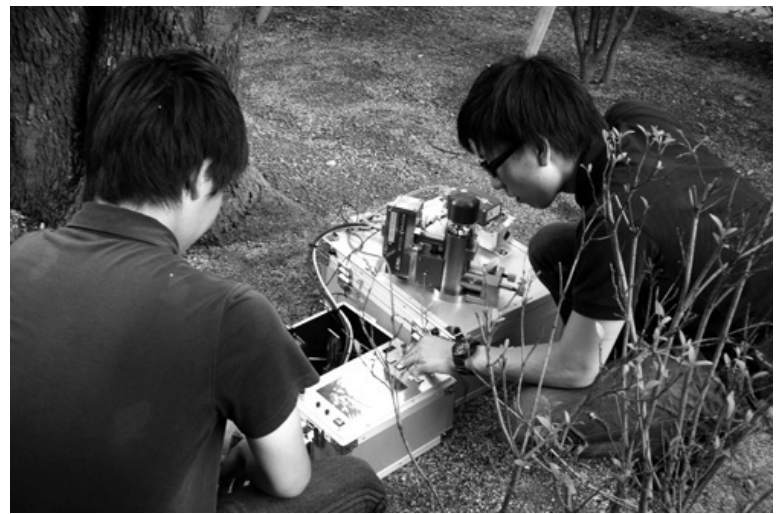

Fig. 1b Observation by MUST-SEM in outdoors.

The carrying-case can be used as a table. The MUST-SEM can be operated for $2 \sim 3$ hours with two battery-packs that is enough to observe more than 10 samples.

1) Observation under low vacuum of $10 \sim 100 \mathrm{~Pa}$ by using a differential pumping system.

2) Optimization of the primary electron energy.

3) Optimization of the incident angle of the primary electron.

4) Coating the sample surface with thin film of conductive material such as metal or carbon.

Techniques of 1) $\sim 3$ ) requires additional function for SEM-column and its controller, leading to the complication and the high cost of the apparatus. The last one generally requires another vacuum apparatus for vacuum evaporation or sputter coating, in addition to the SEM apparatus. Since this is not adequate for mobile-use in outdoors, we employed the ionic liquid 
coating method discovered by Prof. S. Kuwabata et al. in 2006 [2-3].

The ionic liquid is a kind of salt, which is in the liquid phase at room temperature, and does not evaporate and keeps the high ionic conductivity even in the vacuum environment. In the present study, we chose EMI-TFSI $\left(\mathrm{C}_{8} \mathrm{H}_{11} \mathrm{~F}_{6} \mathrm{~N}_{3} \mathrm{O}_{4} \mathrm{~S}_{2}\right)$, which shows a relatively low viscosity among many kinds of ionic liquid and is often used for an electrolyte of a dye-sensitized solar cell and etc. The chemical structure of EMI-TFSI is shown in Fig.2.

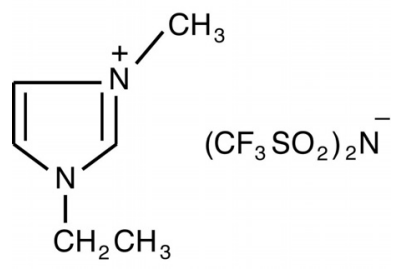

Fig. 2 The chemical structure of EMI-TFSI ionic liquid. The melting point is $-15 \mathrm{deg}$. $\mathrm{C}$, and the flash point is more than 200 deg. C.

\section{Experimental Results}

Non-conductive samples were dipped in the ethanol solution containing a few percent of EMI-TFSI by volume. After evacuation in the SEM-column, ethanol evaporates from the sample surface and the left EMI-TFSI forms the conductive thin film on the sample surface. The acceleration voltage of the primary electron was fixed to $3 \mathrm{kV}$. The typical primary beam current was $60 \sim 100 \mathrm{nA}$.

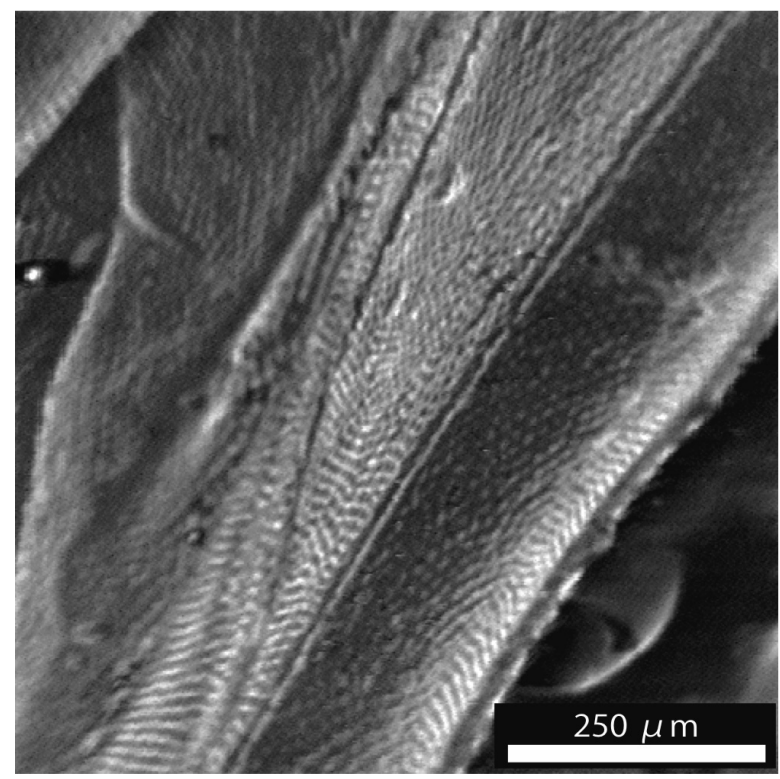

Fig. 3a SEM image of a fly's wing.

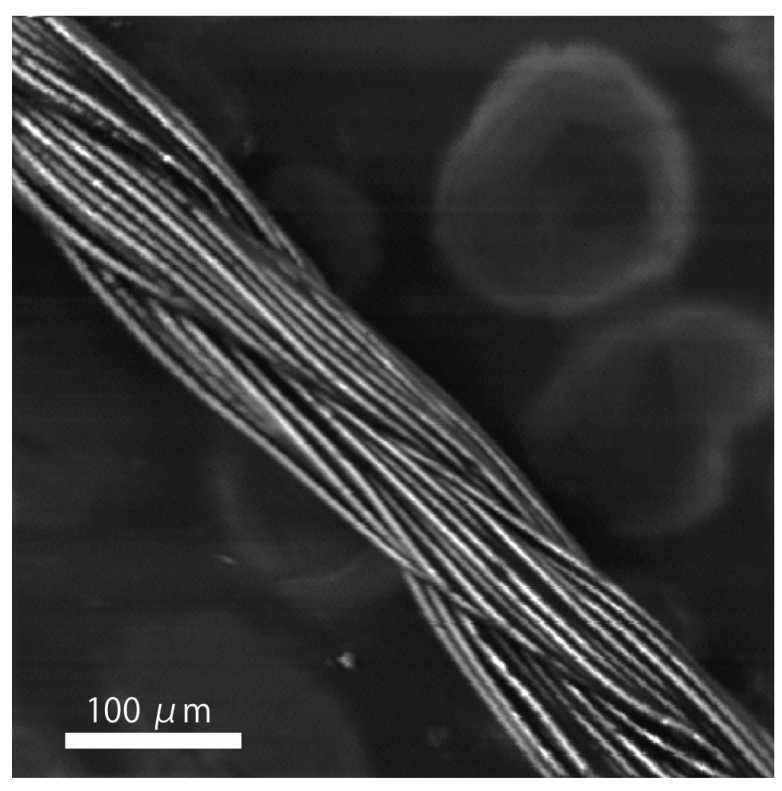

Fig. 3b SEM image of a Polyester sewing thread.

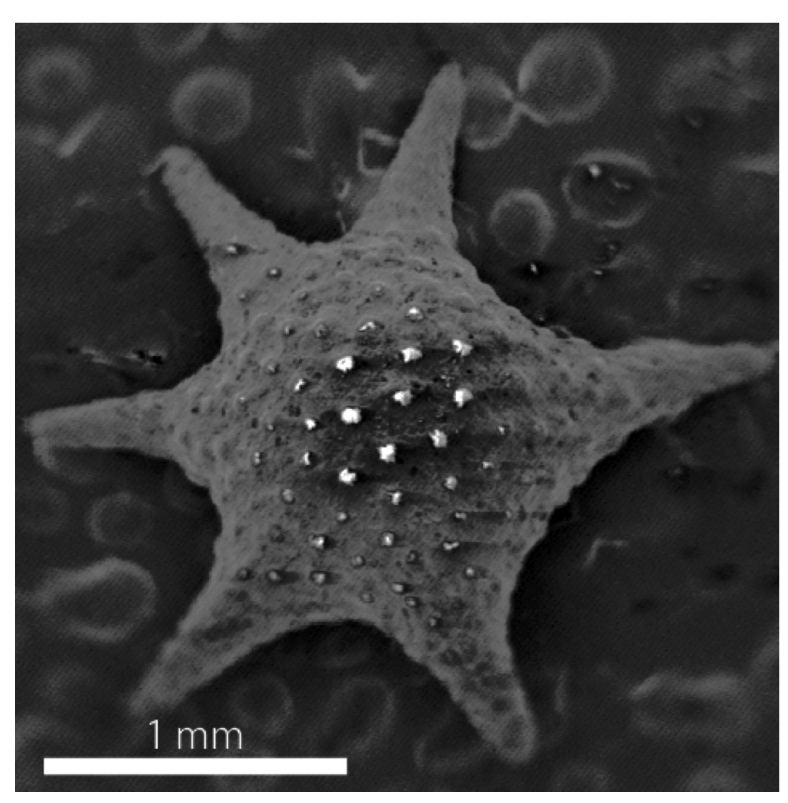

Fig. 3c SEM image of a 'star-sand', a shell of foraminifera Baculogypsina sp. made by $\mathrm{CaCO}_{3}$.

Observation results are shown in Fig.3. These are SEM images of biological samples (a fly's wing in Fig. 3a and intertwined DNA fibers extracted from banana and supported on a metallic mesh in Fig. 3f), a synthesized polymer (a Polyester sewing thread in Fig. $3 b$ ), and natural minerals (shells of foraminifera made by calcium carbonate in Figs. 3c-3d and a microfossil, that is a skeleton of radiolarian made by silica in Fig. $3 e)$. Both of foraminifera and radiolarian are sea plankton.

Since these non-conductive samples are porous or 
have complicated surface topography, they absorb the ionic liquid of low viscosity. Although this might smear out the surface fine structure of less than $1.7 \mu \mathrm{m}$ corresponding to the resolution of the MUST-SEM, all of these samples could be observed clearly, indicating that the ionic liquid coating method is very simple and effective to reduce the charging during the MUST-SEM observation.

\section{Summary}

A battery-powered mobile scanning electron microscope (MUST-SEM) was developed for elementary science education. The ionic liquid coating method was applied to the MUST-SEM observation of non-conductive materials such as biological samples, synthesized polymers and natural minerals. It could be confirmed that the ionic liquid coating method is adequate for the observation of non-conductive materials by the present mobile SEM, MUST-SEM, in outdoors.

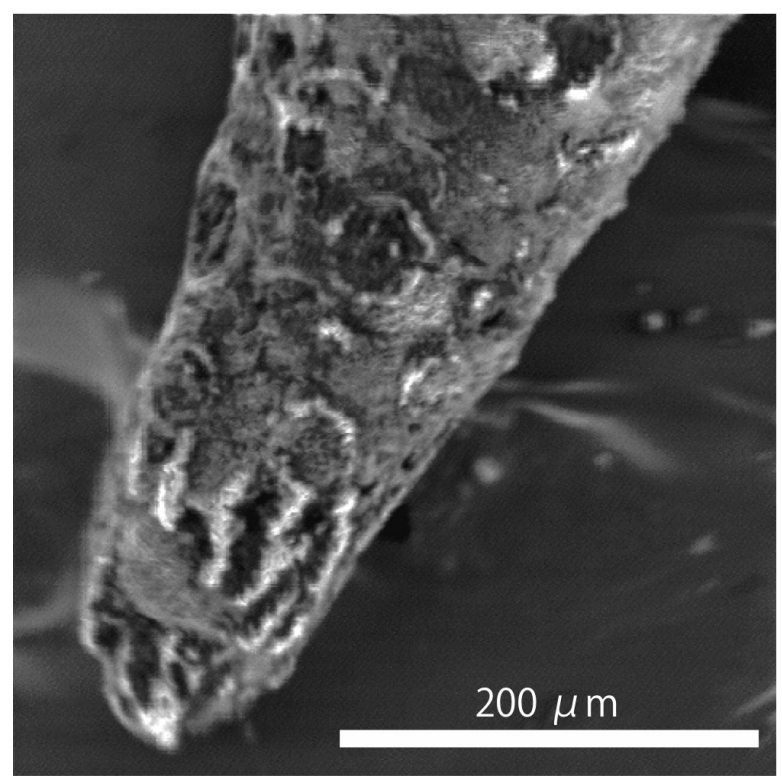

Fig. 3d SEM image of a 'star-sand', a shell of foraminifera Baculogypsina sp. made by $\mathrm{CaCO}_{3}$.

\section{Acknowledgement}

The authors would like to acknowledge the helpful advices of and fruitful discussions with Prof. R. Shimizu of International Institute for Advanced Studies, Drs. K. Tsunoyama and S. Saito of Japan Science and Technology Agency.

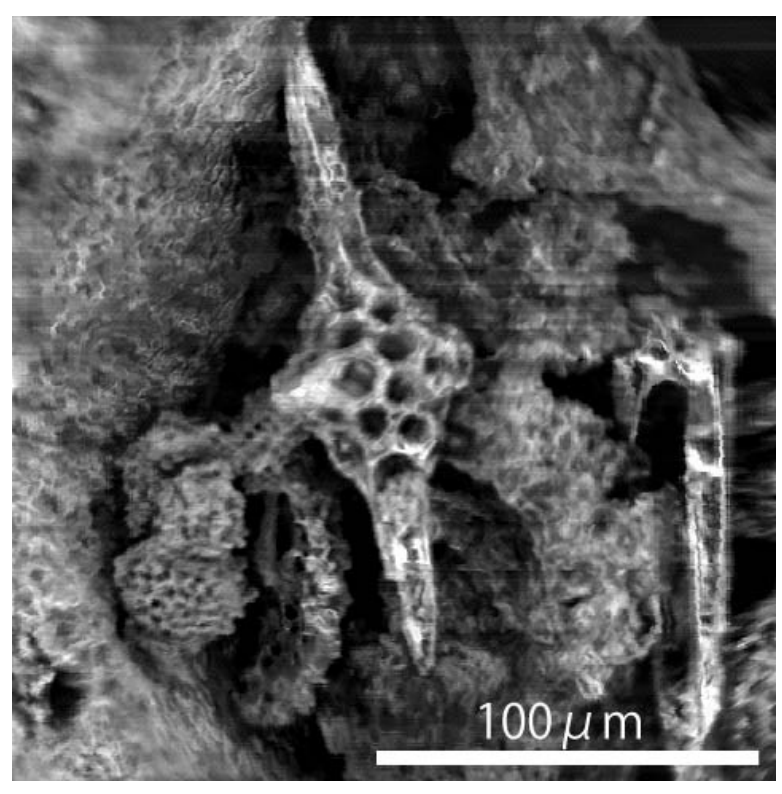

Fig. 3e SEM image of a microfossil of Jurassic radiolarian Trillus sp., a skeleton made by $\mathrm{SiO}_{2}$.

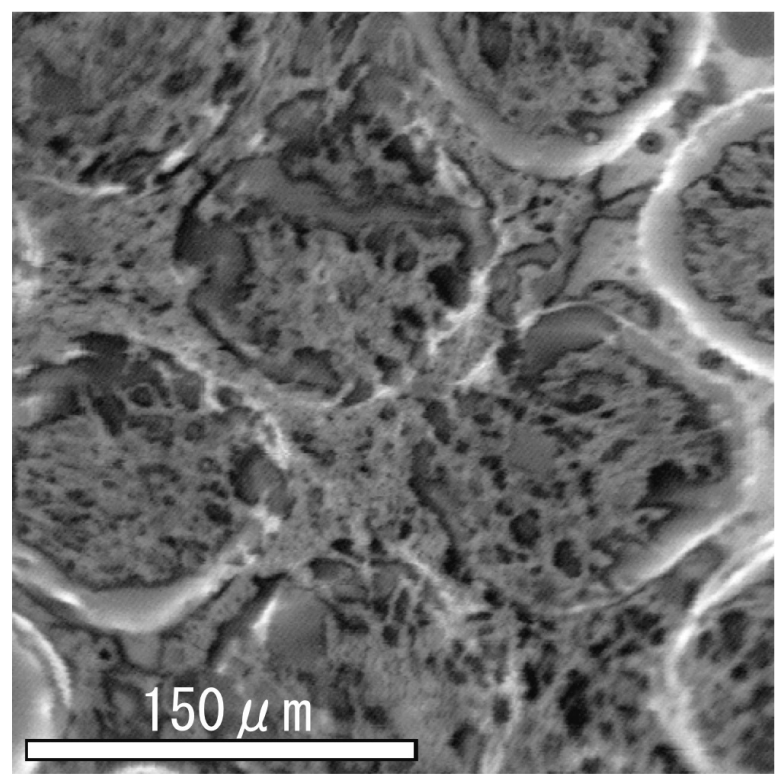

Fig. 3f SEM image of intertwined genomic DNA fibers extracted from banana, supported on a metallic mesh (200 meshes/inch) for TEM.

Thanks are also due to Dr. K. Kuwahara of Kansai University and Prof. M. Yokokawa of Osaka Institute for Technology for providing us the sample of microfossils.

This study was supported by SENTAN, JST (Japan Science and Technology Agency). 
Journal of Surface Analysis Vol. 18 No. 2 (2011) pp. 105-109

M. Inoue et al. Application of Ionic Liquid Coating Method to Observation of Non-conductive Samples by...

\section{References}

[1] M. Inoue, Y. Hashimoto, T. Iyasu, K. Moriguchi, and T. Tanaka, Proceedings of the 7th International Symposium on Atomic Level Characterizations for New Materials and Devices '09 (Hawaii, December 6-11, 2009), p.253-254.

[2] S. Kuwabata, A. Kongkanand, D. Oyamatsu, and T. Torimoto, Chem. Lett. 35, 600-601 (2006).

[3] S. Arimoto, M. Sugimura, H. Kageyama, T. Torimoto, and S. Kuwabata, Electrochimica Acta 53, 6228-6234 (2008). 